four weeks and six months. Joan Kempster, aged 24 years, unmarried, reputed normal.

(10-14) The children of Ann Redding (III, 5), herself affected. (10) Winifred, aged 35, reputed normal. (11) William Redding, aged 33 years, reputed normal. (12) Ann, married Puddephat, aged 31 years; reputed normal. (13) Dorothy, married Blunt, aged 28 years, reputed normal. (14) Percival Redding, aged 26 years, reputed normal.

(15-17) The children of Rose Graves (III, 6), herself affected. All in Canada. Rosemary Graves, aged 29 years, is reputed to be mentally defective; unmarried. Doris Graves, aged 27 years, reputed normal, unmarried. Robert Graves, aged 21 years, reputed normal; was seen in 1939, at the age of 12 years, and was then found normal.

(18) Thomas Wood, the son of Eliza Wood (III, 7), aged 25 years; reputed normal.

(19) Sidney Kempster, son of Joseph Kempster (III, 8), himself affected, aged 28 years, married, no children; reputed normal.

(20-23) The children of Arthur Kempster (III, 9), himself affected. (20)

Herbert, aged 26 years. (21) Fred, aged 24 years. (22) Robert, aged 17 years. (23) John, aged 9 years. All reputed to be normal.

(24 and 25) The children of Robert Kempster (III, 10), Gwen, aged 18 years, and Margaret, aged 14 years, reputed to be normal.

Generation V.

( 1 and 2) Ronald and Josephine Collins, aged 2 and 1 years, children of IV, 2.

(3) Michael Cook, aged 1 year, son of IV, 4.

(4-6) (4) Brenda, aged. 8 years, Anthony, aged 5 years, Nita, aged 2 years, children of William Kempster (IV, 11).

(7-8) Harold and Sandra Puddephat, aged 8 and 6 years respectively, children of IV, 12.

(9) Stephen Blunt, aged 4 months, son of IV, 13.

(10) Stewart Redding, aged 14 months, son of IV, 14.

(11) Margaret Kempster, aged 12 months, daughter of IV, 20.

\title{
A THEORETICAL PLAN OF A. METHOD FOR REMOVING NON-FERRO-MAGNETIC METALLIC INTRA-OCULAR FOREIGN BODIES BY MEANS OF ELECTRO.MAGNETIC FORCES.*
}

BY

\section{P. M. ENDT and J. Ten Doesschate}

UTRECHT

THE removing of non-ferro-magnetic metallic intra-ocular foreign bodies (like copper, non-magnetic steel, etc.), always offers many difficulties to the ophthalmologist. For removing ferro-magnetic foreign bodies we have at our disposal very satisfactory methods which are all based on the principle of exerting electro-magnetic forces on the foreign body. Theoretically, however, it is possible

${ }^{*}$ A paper on this subject was read by the authors at the 112 th meeting of the Netherl. Ophthal. Soc. on June 7, 1947. This paper did not contain the necessary physical foundations. 
to exert electro-magnetic forces also on non-ferro-magnetic bodies. In this paper we propose to give a theoretical development of a method which will allow the removing of such foreign bodies, provided a high frequency electro-magnetic field of sufficient strength can be produced.

First, the principles of the new method will be given qualitatively.

In the metallic particle in question an alternating induction current is generated by a high frequency magnetic field. For simplicity let us assume the particle to be a little ring in a plane at right angles. with the direction of the magnetic field. Now there exists a well-known analogy of a ring current with a magnetic dipole. On a magnetic dipole a force is exerted by an inhomogeneous magnetic field. Analogously also the ring undergoes a force by the magnetic field, provided the alternating current in the ring is in phase with the alternating magnetic field. The latter is true when the frequency is so high, that the inductance of the ring is large compared with its resistance.

Now we will follow this argument quantitatively. Here, however, we will assume the particle to be a little sphere (radius $R$ ).

By an alternating magnetic field $H(t)$ a magnetic dipole is induced in the particle with dipole strength $\left.{ }^{1}\right)$ :

$$
\vec{p}(t)=-\frac{1}{2} R^{3} \vec{H}(t) \quad(\text { e.s.u. }) . \quad \ldots \quad \cdots
$$

This relation is subject to two restrictions. First the frequency $\nu$ must be so low, that the wavelength related with it: $\lambda=c / \nu$ ( $c=$ velocity of light) is large compared with the radius of the particle. This restriction is not at all severe. On the other hand the penetrating depth $d$ of the skin effect must be small compared with the radius:

$$
d=\frac{c}{2 \pi \sqrt{\nu \sigma}} \ll R . \quad \ldots, \quad \ldots
$$

Here $\sigma$ is the electrical conductivity of the particle in e.s.u.

The force exerted on the particle will now be given by:

$$
\vec{F}(t)=\overrightarrow{(p}(t) \operatorname{grad}) \vec{H}(t)=-\frac{1}{4} R^{3} \operatorname{grad}\left(H^{2}(t)\right) \text {. }
$$

This force is still dependent on time. The mean force is given by :

$$
\overrightarrow{\bar{F}}=-\frac{1}{4} R^{3} \operatorname{grad}\left(H_{\sim^{2}}\right), \quad \ldots \quad \ldots
$$

where $H_{\sim}$ is the root mean square value of the magnetic field $H(t)$. Preliminary experiments done by one of us in the Physical Laboratory of the University of Utrecht with a small high-frequency generator show ed that this force has the expected order of magnitude. 
More important than the force itself is the ratio of the electromagnetic force to the force of gravity $(G)$ exerted on the particle :

$$
F / G=\frac{1 / 4 R^{3} \operatorname{grad}\left(H_{\sim}{ }^{2}\right)}{4 / 3 \pi R^{3} \rho g}=\frac{3}{8 \pi} \frac{H_{\sim} \operatorname{grad} H_{\sim}}{\rho g}, \quad \ldots
$$

-where $\rho$ is the density of the particle and $g$ the acceleration of gravity. We see that this ratio is independent of the radius of the particle.

Let us compare equation (3) with the force exerted on an iron sphere (permeability $\mu$ ) with the same radius $R$ by a constant magnetic field. This force is given by:

$$
\vec{F}=\frac{1}{2} \frac{\mu-1}{\mu+2} R^{3} \operatorname{grad}\left(H^{2}\right) . \quad \ldots \quad \ldots
$$

With $\mu \gg 1$ we have :

$$
\vec{F}=\frac{1}{2} R^{3} \operatorname{grad}\left(H^{2}\right) . \quad \ldots \quad \ldots
$$

Comparing (3) and (5) we note that the force on a copper particle in an alternating field is identical with that on a diamagnetic sphere $(\mu<<1)$ in an equal constant field.

Comparing (3) and (6) we see that if $H_{\sim}=H$ there remain two differences :

1 -The force on the copper sphere is one half of that exerted on the iron sphere. This is not serious.

2-The force in (3) has the minus sign. Whereas the iron particle is drawn into the coil producing the magnetic field, the copper particle is pushed out of it. This is rather a serious disadvantage. To extract particles out of the eye the coil must be held behind the head. The distance from the coil to the eye now being large, the alternating current in the coil must be very strong to produce a magnetic field of sufficient strength.

Another unwanted effect is the generation of heat in the particle by the induction current, given by ${ }^{1}$ ) :

$$
W=\frac{3}{4} R^{2} H_{\sim}^{2} c \sqrt{\nu / \sigma} \text { erg/sec . } \quad \ldots \quad \ldots
$$

The heat conductivity of the ocular fluids being $\Lambda$, the temperature excess of the particle becomes:

$$
\theta=\frac{W}{4 \pi R \Lambda}=\frac{3 R H_{\sim}^{2} c \sqrt{\nu / \sigma}}{16 \pi}
$$

If the particle were to become too hot, albumen in the ocular fluids would coagulate around the particle.

The force of the particle being, between the limits given, independant of the frequency, it is clear that we will use a frequency as low 
as possible in order not to heat the particle excessively. Let us choose the frequency so that the penetrating depth of the skin effect (see (2) ) equals one half the radius of the particle:

$$
d=\frac{c}{2 \pi \sqrt{\nu \sigma}}=\frac{1}{2} R . \quad \ldots \quad \ldots
$$

The temperature excess now becomes independent of the radius :

$$
\theta=\frac{3 c^{2}}{16 \pi^{2} \sigma \Lambda} H_{\sim^{2}}^{2} \quad \ldots \quad \ldots
$$

As an example we will consider an aluminium particle $(\rho=2 \cdot 7$, $\sigma=3.4 \times 10^{17}$ e.s.u.) of arbitrary radius. We take the heat conductivity of the ocular fluids as equal to that of pure water: $\Lambda=6 \times 10^{4}$ $\mathrm{erg} / \mathrm{sec} \mathrm{cm}{ }^{\circ} \mathrm{C}$. If the magnetic field has an r.m.s. value of 300 Oerstedt and the gradient of the $r . m . s$. field is 100 Oerstedt $/ \mathrm{cm}$., then we have from (4) and (10) $\left(c=3 \times 10^{10} \mathrm{~cm} / \mathrm{sec}, g=981 \mathrm{~cm} / \mathrm{sec}^{2}\right)$ :

$$
F / G=1.34 \text { and }: \theta=74^{\circ} \mathrm{C} \text {. }
$$

The optimum frequency given by (9) becomes :

$$
\nu=\frac{c^{2}}{\pi^{2} R^{2} \sigma} \cdot \quad \cdots \quad \ldots \quad \cdots
$$

For an aluminium particle with $R=1 \mathrm{~mm}$. we have : $\lambda=26 \mathrm{kHz}$.

For copper particles the temperature excess is larger, if $F / G$ is kept constant; the electrical conductivity is larger but the density is also larger.

It may be necessary to operate the magnetic field only during short time-intervals in order to keep down the temperature of the particle.

\section{Summary}

A theoretical development is given of a method which will allow the removal of non-ferro-magnetic metallic intra-ocular foreign bodies by means of a high-frequency electro-magnetic field, provided a field of sufficient intensity can be produced and the difficulties connected with heat generation can be overcome.

The authors wish to express their sincere thanks to Professor Dr. J. M. W. Milatz for his lively interest in this work.

\section{REFERENCE}

1. CASimiR, H. B. G. (1947).-Philips Research Report, 2, 42. 